\title{
POPULATIONS IN THE Z=0.23 RICH CLUSTER ABELL 2390
}

\author{
J.B.HUTCHINGS, R.ABRAHAM, T.SMECKER-HANE \\ Dominion Astrophysical Observatory, Victoria, B.C., Canada \\ S.MORRIS, T.DAVIDGE, M.RIGLER \\ Dominion Astrophysical Observatory, Victoria, B.C., Canada \\ AND \\ R.CARLBERG, H.YEE, E.ELLINGSON \\ University of Toronto, Canada; University of Colorado, USA
}

\section{Summary}

We have spectra, colours and morphologies from the CFHT for 240 cluster members and 80 field galaxies over a $7 \times 46$ arcmin field. The cluster galaxies show strong radial gradients in colour, morphology, and spectrum. The central group has only red early-type galaxies of high central concentration, which must have formed at least $5 \mathrm{~Gy}$ ago. The population becomes bluer and shows an increasing fraction of disk morphology and recent star-formation with clustocentric radius, and eventually blends into the field. However, the fraction of blue galaxies is significantly lower than in Butcher-Oemler clusters, and there are only two starburst galaxies (one of which is the cluster $\mathrm{cD}$ ). Fitting of line measures with stellar population models indicates that star-formation has been truncated in the $15-20 \%$ of the cluster galaxies that have strong $\mathrm{H} \delta$ absorption, and that these galaxies are not all blue. There is evidence that cluster galaxies are dusty compared with the field. The morphology gradient can be fit with models of disk fading. However, there is a small fraction of interacting and merging galaxies, which must play a part in the population evolution. The cluster is accreting from the field in a non-violent way, including some distinct subgroups. This is different from what is seen at both higher and lower redshift, and if generally true, suggests a high Omega universe.

These results are being published in detail elsewhere. 